\title{
Treatment of Rapid Progression of Myopia: Case Series and Literature Review
}

\author{
Nir Erdinest ${ }^{a}$ b Naomi London ${ }^{c} \quad$ Nadav Levinger ${ }^{a, d}$ Itay Lavy ${ }^{a}$ \\ Eran Pras ${ }^{\mathrm{e}}$ Yair Morad ${ }^{\mathrm{b}}$ e \\ aDepartment of Ophthalmology, Hadassah-Hebrew University Medical Center, Jerusalem,

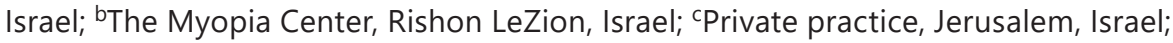 \\ ${ }^{\mathrm{d} D e p a r t m e n t}$ of Opthalmology, Enaim Refractive Surgery Center, Jerusalem, Israel; \\ eDepartment of Ophthalmology, Assaf Harofeh Medical Center, Zerifin, Israel
}

\author{
Keywords \\ Myopia progression · Myopia control · Atropine · Peripheral defocus · Soft contact lenses
}

\begin{abstract}
This retrospective case series demonstrates the combination of $0.05 \%$ atropine with MiSight ${ }^{\circledR}$ 1 day (Cooper vision, Sar Ramon, CA, USA) in rapid progression of myopia of 4 children. MiSight ${ }^{\circledR} 1$ day is a peripheral defocus, center-distance soft contact lens and is effective at controlling moderate progression of myopia during the course of 1 year. The current case series included 2 females and 2 males with an average age of $9.68 \pm 0.26$ years and an average axial length of $24.81 \pm 0.92 \mathrm{~mm}$. Their myopic progression during the previous year was -1.45 $\pm 0.27 \mathrm{D}$. The children had not attempted any myopia control thus far. This relatively high increase prompted a combination treatment of daily instillation of $0.05 \%$ atropine and MiSight, a daily replacement soft contact lens. Cycloplegic refraction and a slit-lamp evaluation were performed every 6 months to confirm no adverse reactions or staining was present. The 8-item contact lens dry eye questionnaire (CLDEQ-8) score of these children was $10.66 \pm 1.52$. The average myopia progression at the end of 1 year decreased to $-0.41 \pm 0.11 \mathrm{D}$, and the average axial length increase was $0.28 \pm 0.08 \mathrm{~mm}$. To the best of the authors' knowledge, this is the first published study showing a combination of $0.05 \%$ atropine and peripheral defocus soft contact lenses indicating efficacy at controlling moderate myopia progression.
\end{abstract}

(C) 2021 The Author(s).

Published by S. Karger AG, Basel 
Erdinest et al.: Treatment of Rapid Progression of Myopia

\section{Introduction}

Myopia is increasing globally in prevalence and magnitude, influenced both by genetic and environmental factors, shown to be interconnected [1-4]. Acknowledged potential myopia-induced pathologies such as retinal detachment, macular degeneration, glaucoma, and cataract have prompted considerable research in an attempt to prevent progression [2-4].

To date, the current options include atropine, peripheral myopic defocus including orthokeratology and soft bifocal or multifocal center-distance contact lenses, increased sunlight, and bifocal or progressive addition spectacle lenses [1]. While each has demonstrated effectiveness in specific cases, the possible additive effect of combining therapies is just beginning to be explored. This case series demonstrates the potency of a combination of $0.05 \%$ atropine and specially designed peripheral defocus soft contact lenses in children with rapidly progressing myopia over a period of 1 year.

\section{Case Presentation}

Methods

Presented here is a retrospective case series of 4 children, 2 females and 2 males with no common genealogy, between the ages of 9.3 and 9.9 years (average $9.68 \pm 0.26$ ) with progressing myopia. The initial exam presented a cycloplegic spherical equivalent refraction (SER) of -2.00 to $-4.75 \mathrm{D}$ of myopia (average $3.72 \pm 1.00 \mathrm{D}$ ) with less than or equal to $1.25 \mathrm{D}$ of astigmatism, measured using a trial frame under monocular as well as binocular conditions.

The average myopia progression the year prior to this appointment was $-1.45 \pm 0.27 \mathrm{D}$ (Table 1). The children habitually spent an average of half an hour outside daily, and none of them reported participating in any extracurricular sport activities. One of the parents has myopia above $3.00 \mathrm{D}$. Understanding the possible environmental influences of myopia progression, they were eager to try and control the rapid increase in their offspring.

Various treatment options were discussed with the parents and the children, including atropine drops (low-dose $0.01 \%$ or $0.05 \%$ ) either in combination or in isolation, progressive addition spectacle lenses, orthokeratology, or soft peripheral defocus contact lenses. The potential risks and benefits related to each option were discussed, and published data were presented and explained. They chose to combine $0.05 \%$ atropine with periphery defocus soft contact lenses.

These children had no previous treatment for myopia control nor did they have any experience with contact lens. The children's binocular status indicated no amblyopia, strabismus, nor accommodative imbalances or oculomotor dysfunctions. Near point of convergence was measured to be within $5 \mathrm{~cm}$ of the nose for all. One child exhibited a $2^{\wedge}$ near point esophoria, and the other 2 exhibited orthophoria, measured using the alternating cover test with an accommodative target at $6 \mathrm{~m}$ and $40 \mathrm{~cm}$.

The children had no systemic preconditions that would affect lens wear such as diabetes or autoimmune conditions. None presented with any contraindications to contact lens wear, nor did they have dry eyes or any tarsal papillae. Their horizontal visible iris diameters (HVID) were between 11.6 and $11.9 \mathrm{~mm}$ (Avg $11.8 \pm 0.11$ ), and the mean corneal keratometry was $7.71 \pm 0.08 \mathrm{~mm}$. MiSight ${ }^{\circledR}(1$ day; Cooper Vision, USA) spherical single-use soft lenses made of omafilcon A were prescribed. They consist of a 14.20-mm diameter and an abase curve of $8.7 \mathrm{~mm}$. The lens design incorporates 2 sets of alternating concentric rings of more center distance and $\mathrm{a}+2.00 \mathrm{D}$ addition ring surround. They were instructed to be worn at least $10 \mathrm{~h}$

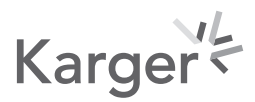




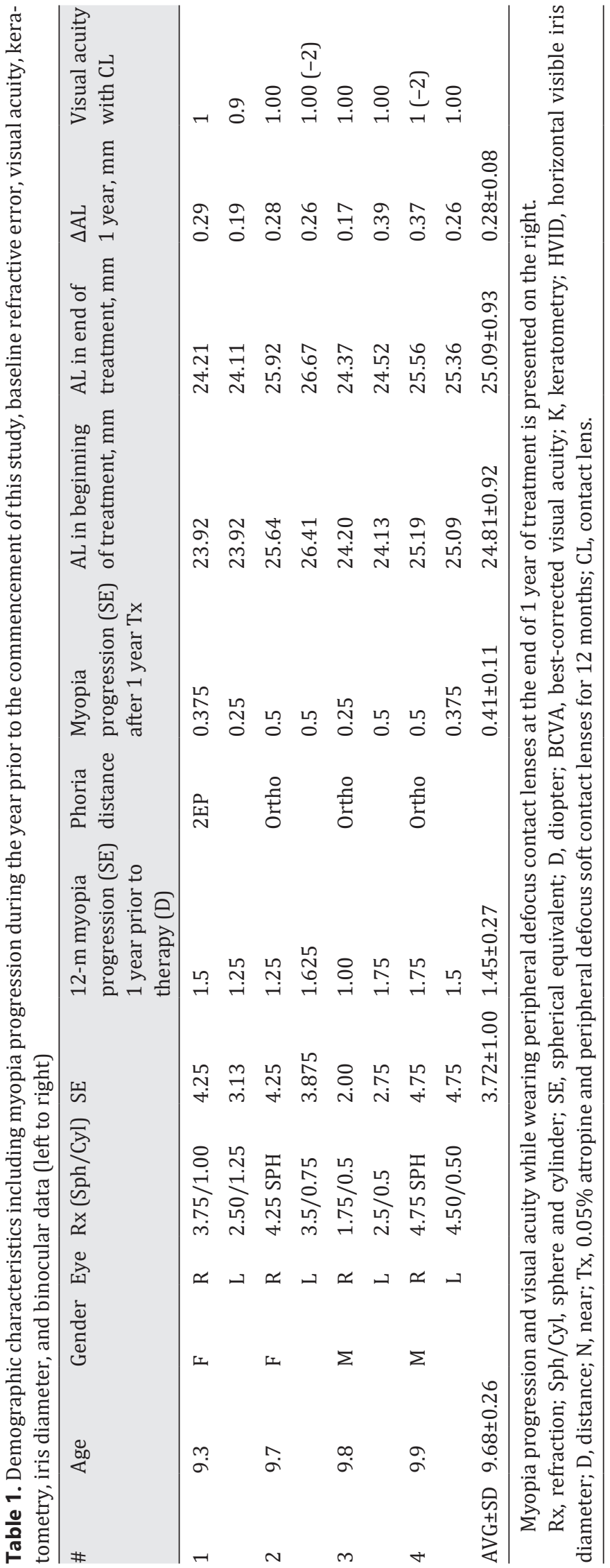


per day 6 days a week. The lens prescription given was the full spherical equivalent refractions, adjusted for vertex distance as required.

The lenses were well centered, and movement upon blink was 1-2 mm and presented minimal movement on peripheral gaze. The parents and children were instructed on insertion and removal of the lenses as well as proper handling. The children stated the lenses were comfortable, and their objective visual acuity (VA) was not inferior to that obtained with their spectacles.

Concurrently, the children were instructed to instill 1 drop of $0.05 \%$ atropine in each eye daily before bedtime. This was prepared by a pharmacy chain (Super-Pharm Professional, Petach-Tikva, Israel) and packaged in opaque (to protect from photodegrading), $10.0 \mathrm{~mL}$ sterile bottles with $5 \mathrm{~mL}$ volume preserved with benzalkonium chloride $0.05 \%$. The atropine sulfate bottles were stored up to 21 days in $4^{\circ} \mathrm{C}$.

Follow-up visits were conducted at 1 week, 1 month, and 3 months after dispensing, establishing ocular health and absence of adverse reactions. A slit-lamp evaluation and cycloplegic refraction were conducted bi-annually to ensure ocular health. They were instructed not to wear the contact lenses for the $24 \mathrm{~h}$ prior to examination. Refraction was measured after instillation of 2 drops of tropicamide 1\%, 1 drop at 5-min intervals.

The SER measurements were performed after mydriasis by 1 practitioner in 1 examination room using identical ambient lighting. Distance VA was measured monocularly using a LogMar chart.

\section{Results}

During the year prior to commencement of treatment, the increase in myopia was between an average of $1.45 \pm 0.27 \mathrm{D}$. After 1 year of the therapy, the average change in the SER measured was $-0.41 \pm 0.11 \mathrm{D}$, and the average change in the axial length was $0.28 \pm 0.08 \mathrm{~mm}$.

Binocular status and visual acuity did not change during the year. The anterior ocular surface examination with fluorescein through a yellow filter and lissamine green through a red filter ensured there were no corneal erosions, abrasions, or staining on the conjunctiva. The tarsal conjunctiva was examined to rule out signs of papillary conjunctivitis, and there was no noted tarsal or bulbar conjunctival erythema. None of the children suffered from glare, nor did they experience difficulty with near vision. Their score of the 8-item contact lens dry eye questionnaire (CLDEQ-8, scored 0-37, higher score is worse) was $10.66 \pm 1.52$. If necessary, the children were assisted by the parents to complete the questionnaire. The CLDEQ-8 scores reflected the children were overall satisfied with contact lens wear [5].

\section{Discussion}

This case series points to the efficacy of combining $0.05 \%$ atropine and peripheral defocus soft contact lens at myopia control. The myopia increase of these children declined from an average of $-1.45 \pm 0.27 \mathrm{D}$ the year prior to treatment to an average of $-0.41 \pm 0.11 \mathrm{D}$ at the end of the year, exhibiting a pronounced decrease of more than two-thirds of progression.

Myopia progression of children wearing MiSight lenses as a monotherapy over the course of 1 to 3 years has been researched. The largest study in the published literature to date followed 109 children of varying rates of progression in which the average increase in myopia after year 1 was $-0.27 \pm 0.07 \mathrm{D}$ which represented a reported average decrease of $50 \%$ in those children. In this same study, potency did not improve during the second and third year, but was still more effective than the control group undergoing no treatment [6].

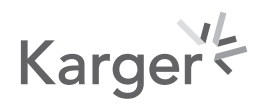


Atropine has been shown to be proficient in many studies, specifically $0.05 \%$ had been acknowledged in the literature at the commencement of this study to be both an effective monotherapy at decreasing myopia progression combined with a relatively low rebound effect after cessation of instillation, more so than an $0.01 \%$ concentration when considering patients with a moderate axial progression $[7,8]$. This concentration was shown in the LowConcentration Atropine for Myopia Progression (LAMP) study to be the most potent, exhibiting a progression of $-0.27 \pm 0.61 \mathrm{D}$ over a 1 -year period. It should be noted that these data comprised a group of children with varying rates of progression, and of this same group $28 \%$ still had an increase of myopia between -0.50 and $2.00 \mathrm{D}$ at the end of 1 year $[2,7,8]$. This study further noted comparable though not added efficacy in the second year [2,9]. The effect of age may be an influencing factor on individual response as discussed in a secondary analysis of the LAMP study [10]. Though the children in all age groups (from age 4 to 12) tolerated various low concentrations well, the very young children were associated with a lower response. The children in the series depicted here correspond to an age group that exhibited a strong response, which was also dose dependent [10]. A clinical trial investigating combining $0.05 \%$ atropine with orthokeratology is underway, but to date, no data have been published as to the possible added efficacy nor that of any other treatment combined with $0.05 \%$ atropine [11].

The Bifocal Lenses In Nearsighted Kids (BLINK) study, launched in 2017, compared identical design single-vision, center-distance soft multifocal contact lenses with a $+1.50 \mathrm{D}$ addition to $\mathrm{a}+2.50 \mathrm{D}$ addition for myopia control [12]. The results so far suggest that there may be a either a dioptric threshold or a minimal area of visual field required of blur to achieve the inhibitory effect $[3,4]$. This supports using a lens such as the MiSight which incorporates 2 concentric circles of peripheral blur thereby providing a larger retinal area of peripheral defocus as well as assuring some blur in both photopic and scotopic circumstances.

Higher doses of atropine for myopia control such as $1 \%$ have been prescribed for myopia control and have shown to be very effective. These concentrations frequently presented more side effects such as photophobia and near blur, often resulting in noncompliance or dropout [13]. A multi-faceted approach such as incorporating a peripheral blur contact lens could perhaps both help with these side effects and control myopia from another angle. Studies have begun publishing effectiveness found after part-time instillation of $1 \%$ atropine (weekly, monthly, and 1-3 times per week) showing promising results $[13,14]$. As data continue to emerge, myopia control will ultimately be more individually adjusted to achieve best outcomes.

Understanding the multifactorial nature of myopia imposes a challenge to effective control. Current treatments primarily target the biological or physiological components. Though the precise mechanisms of action of atropine remain elusive, the proposed options are 5 -fold. Atropine is a reversible competitive antagonist, with a nonselective affinity for all subtypes of the muscarinic receptors in the sclera that cause proliferation of fibroblasts and axial elongation. Atropine has been shown to reduce epidermal growth factor receptor activity in scleral fibroblasts and to inhibit the choroidal thinning induced by hyperopic retinal defocus. It increases the neurotransmitter dopamine release, which prevents ocular eye growth. Last, atropine intervenes in other biological processes including the retinal signaling pathways that respond to environmental cues as well as in the retinal pigment epithelium when relaying ocular growth regulatory signals from the retina to sclera [15].

The mechanism underlying the positive influence of optical peripheral myopic defocus is not understood. Hypotheses include reducing the accommodative lag [16] and possibly also suspending the excessive expansion of Bruch's membrane [17]. Still undetermined are the exact location on the retina, the surface area required, or the depth of myopic defocus needed for maximum efficacy.

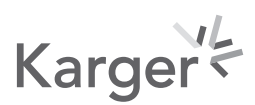


In summation, the objective was to attempt to control the progression using 2 different techniques. The compound optical (peripheral defocus contact lens) and biological (atropine) components complement each other and potentially may prove more effective than when administered in isolation.

Larger cohort randomized double-blind studies over a few years would clarify whether this modality is effective to the same degree or perhaps improves with time. Possibly a different schedule of contact lenses wear would positively affect the outcome. Additional research would help determine when an adjustment to the atropine concentration is required in different cases of myopia progression.

\section{Conclusions}

Combination of $0.05 \%$ atropine and peripheral defocus daily replacement soft contact lenses exhibited high effectiveness at controlling myopia during 1 year of treatment in children exhibiting a moderate progression.

\section{Acknowledgments}

Dr. Nir Erdinest is grateful to the Azrieli Foundation for the award of Postdoc Azrieli Fellowship and the Hadassah-Hebrew University Medical Center for the award of postdoctoral fellows.

\section{Statement of Ethics}

Written informed consent was obtained from the legal guardians of the patients for publication of this case. This report does not contain any personal information that could lead to the identification of the patients. This study protocol was reviewed and approved by the Medical Center Institutional Review Board (IRB), Approval No. HMO 0354-21.

\section{Conflict of Interest Statement}

None of the authors of this manuscript have any conflicts of interest to declare.

\section{Funding Sources}

This manuscript did not receive any funding.

\section{Author Contributions}

N.E., Y.M., and I.L. contributed to conceptualization. N.E. and Y.M. treated the patients and collected the clinical data. N.E. and N.L. contributed to original manuscript preparation. N.L, I.L., and E.P. provided comments on previous versions of the manuscript. All authors approved the final version of the manuscript. All authors attest that they meet the current ICMJE criteria for authorship. 


\section{Data Availability Statement}

All data generated during this study are available in Table 1. Further enquiries can be directed to the corresponding author.

\section{References}

1 Mak CY, Yam JC, Chen LJ, Lee SM, Young AL. Epidemiology of myopia and prevention of myopia progression in children in East Asia: a review. Hong Kong Med J. 2018;24(6):602-9.

2 Yam JC, Jiang Y, Tang SM, Law AKP, Chan JJ, Wong E, et al. Low-concentration atropine for myopia progression (LAMP) study: a randomized, double-blinded, placebo-controlled trial of $0.05 \%, 0.025 \%$, and $0.01 \%$ atropine eye drops in myopia control. Ophthalmology. 2019;126(1):113-24.

3 Tang WC, Leung M, Wong ACK, To C-h., Lam CSY. Optical interventions for myopia control. In: Ang M, Wong T, editors. Updates on myopia. Singapore: Springer; 2020. p. 289-305.

4 Walline JJ, Lindsley KB, Vedula SS, Cotter SA, Mutti DO, Ng SM, et al. Interventions to slow progression of myopia in children. Cochrane Database Syst Rev. 2020;1(1):CD004916.

5 Chalmers RL, Begley CG, Moody K, Hickson-Curran SB. Contact lens dry eye questionnaire-8 (CLDEQ-8) and opinion of contact lens performance. Optom Vis Sci. 2012;89(10):1435-42.

6 Chamberlain P, Peixoto-de-Matos SC, Logan NS, Ngo C, Jones D, Young G. A 3-year randomized clinical trial of MiSight lenses for myopia control. Optom Vis Sci. 2019;96(8):556-67.

7 Khanal S, Phillips JR. Which low-dose atropine for myopia control? Clin Exp Optom. 2020;103(2):230.

8 Zhao C, Cai C, Ding Q, Dai H. Efficacy and safety of atropine to control myopia progression: a systematic review and meta-analysis. BMC Ophthalmol. 2020;20(1):478-8.

9 Chia A, Lu QS, Tan D. Five-year clinical trial on atropine for the treatment of myopia 2: myopia control with atropine 0.01\% eyedrops. Ophthalmology. 2016;123(2):391-9.

10 Li FF, Zhang Y, Zhang X, Yip BHK, Tang SM, Kam KW, et al. Age effect on treatment responses to $0.05 \%, 0.025 \%$, and $0.01 \%$ atropine: low-concentration atropine for myopia progression study. Ophthalmology. 2021 Aug; 128(8):1180-87.

11 Tan Q, Ng AL, Cheng GP, Woo VC, Cho P. Combined atropine with orthokeratology for myopia control: study design and preliminary results. Curr Eye Res. 2019;44(6):671-8.

12 Walline JJ, Gaume Giannoni A, Sinnott LT, Chandler MA, Huang J, Mutti DO, et al. A randomized trial of soft multifocal contact lenses for myopia control: baseline data and methods. Optom Vis Sciofficial Publication Am Acad Optom. 2017;94(9):856.

13 Zhu Q, Tang Y, Guo L, Tighe S, Zhou Y, Zhang X, et al. Efficacy and safety of $1 \%$ atropine on retardation of moderate myopia progression in Chinese school children. Int J Med Sci. 2020;17(2):176.

14 Foo LL, Htoon H, Farooqui SZ, Chia A. Part-time use of $1 \%$ atropine eye drops for prevention of myopia progression in children. Int Ophthalmol. 2020;40(7):1857-62.

15 Upadhyay A, Beuerman RW. Biological mechanisms of atropine control of myopia. Eye Contact Lens. 2020; 46(3):129.

16 Sankaridurg P. Contact lenses to slow progression of myopia. Clin Exp Optom. 2017;100(5):432-7.

17 Wu PC, Chuang MN, Choi J, Chen H, Wu G, Ohno-Matsui K, et al. Update in myopia and treatment strategy of atropine use in myopia control. Eye. 2019;33(1):3-13. 